\title{
A photopolymerized composite hydrogel and surgical implanting tool for a nucleus pulposus replacement
}

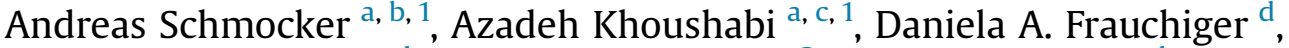 \\ Benjamin Gantenbein ${ }^{\mathrm{d}}$, Constantin Schizas ${ }^{\mathrm{e}, 2}$, Christophe Moser ${ }^{\mathrm{b}}$, \\ Pierre-Etienne Bourban ${ }^{c}$, Dominique P. Pioletti ${ }^{\text {a, * }}$ \\ ${ }^{a}$ Laboratory of Biomechanical Orthopedics, Institute of Bioengineering, École Polytechnique Fédérale de Lausanne (EPFL), Switzerland \\ ${ }^{\mathrm{b}}$ Laboratory of Applied Photonics Devices, Institute of Microengineering, EPFL, Switzerland \\ ${ }^{c}$ Laboratory of Polymer and Composite Technology, Institute of Materials, EPFL, Switzerland \\ d Tissue and Organ Mechanobiology, Institute for Surgical Technology and Biomechanics, University of Bern, Bern, Switzerland \\ ${ }^{\text {e }}$ Centre Hospitalier Universitaire Vaudois, Orthopedic Department, Lausanne, Switzerland
}

\section{A R T I C L E I N F O}

\section{Article history:}

Received 10 September 2015

Received in revised form

6 February 2016

Accepted 15 February 2016

Available online 21 February 2016

\section{Keywords:}

Medical device

poly(ethylene-glycol)dimethacrylate

Minimally invasive surgery

Nano-cellulose fibers

Orthopedic implant

Photopolymerization

\begin{abstract}
A B S T R A C T
Nucleus pulposus replacements have been subjected to highly controversial discussions over the last 40 years. Their use has not yet resulted in a positive outcome to treat herniated disc or degenerated disc disease. The main reason is that not a single implant or tissue replacement was able to withstand the loads within an intervertebral disc. Here, we report on the development of a photo-polymerizable poly(ethylene glycol)dimethacrylate nano-fibrillated cellulose composite hydrogel which was tuned according to native tissue properties. Using a customized minimally-invasive medical device to inject and photopolymerize the hydrogel insitu, samples were implanted through an incision of $1 \mathrm{~mm}$ into an intervertebral disc of a bovine organ model to evaluate their long-term performance. When implanted into the bovine disc model, the composite hydrogel implant was able to significantly re-establish disc height after surgery $(\mathrm{p}<0.0025)$. The height was maintained after 0.5 million loading cycles $(\mathrm{p}<0.025)$. The mechanical resistance of the novel composite hydrogel material combined with the minimally invasive implantation procedure into a bovine disc resulted in a promising functional orthopedic implant for the replacement of the nucleus pulposus.
\end{abstract}

() 2016 Elsevier Ltd. All rights reserved.

\section{Introduction}

The impact of low back pain on society is tremendous [1]. The economic burden was estimated to be up to 355 \$ per capita and annum for direct costs and $507 \$$ for indirect costs [2] which results in a total of 200 billion per annum for a country such as the United States of America [3]. In its early stage, low back pain with or without sciatica is addressed with conservative treatments such as physical therapy or medication [4,5]. In a later stage, decompression surgery might be warranted [6] and in the case of disc

\footnotetext{
* Corresponding author. EPFL/STI/IBI/LBO, Station 19, 1015, Lausanne, Switzerland.

E-mail address: dominique.pioletti@epfl.ch (D.P. Pioletti).

1 Equal contributions.

2 The author's actual address is: Neuro-orthopedic Spine Unit, Clinic Cecil, Lausanne, Switzerland.
}

herniation a more invasive procedure such as discectomy is undertaken [7]. For persisting pain due to disc degeneration fusion of one or several spinal segments might become necessary [8]. The reasons for more severe surgery are mainly disc protrusion and herniation, degenerative disc disease [9] and spondylolisthesis [10]. For all these conditions, the range of motion of the spinal segment increases which leads to a segment instability and pain [11].

The ideal solution is a stabilization of the joint by reinforcing the degenerated or missing tissue of the nucleus pulposus (NP) [12] which is the core of the intervertebral disc (IVD). However, up to date, all implantation attempts with a NP replacement material have failed due to extrusion, expulsion or subsidence of the implanted material $[13,14]$. The required material properties have been subject of controversial discussions over the last two decades. The current, general consensus is that more mechanically resistant materials need to be developed [15-17]. What is clear, is that a material needs to be implanted in a minimally invasive manner to 
avoid damaging existing tissue [15]. It also needs to re-establish disc height without destroying the endplate $[12,16]$ and should not extrude when the spinal segment is cyclically loaded $[13,18]$. We propose an implant solution based on a photopolymerizable material. The low viscosity of the material is ideal for injecting it through a small capillary and for flowing into tissue interstices. The capillary also guides the curing light, thus providing an original solution for minimal intrusion and optimal control.

Photopolymerized implants first appeared more than 40 years ago in dentistry [19] where the initiation of the photopolymerization reaction by light was a significant advantage in terms of control and integration into enamel. Recent advances in water-based and biocompatible materials which allows photopolymerization of volumes of several $\mathrm{cm}^{3}$, open a new avenue for in situ photopolymerized implants for orthopedics [20], oncology [21] and ophthalmology [22]. Especially, photopolymerized hydrogels provide promising solutions for tissue replacements [23]. However, despite the large body of work in photopolymerized hydrogels, none of them to our knowledge, has the performance required for a NP replacement, and there are no devices available for controlled, minimally invasive placement of photopolymerized hydrogels.

Hydrogels lack structural strength, and thus are not adapted to cases of high mechanical stress, such as in the IVD [24]. Conventional methods to improve the hydrogel stiffness, such as increasing the cross-linker density, usually result in a loss of strength and water content [25-27]. Different hydrogel designs have been proposed to tackle this issue $[25,28]$ such as slip-link hydrogels [29] and double network hydrogels [30]. Although these methods result in significant improvement of stiffness and toughness, their long and/or sequential preparation makes in situ curing impractical. The use of a composite hydrogel is an alternative approach. Composite hydrogels combine the high stiffness and strength while preserving the one-step hydrogel preparation. The composite material retains the short curing time making the composite material suitable for in situ insertion.

Minimally invasive photopolymerization has been achieved in situ by transdermal illumination [31] and irradiation through the walls of blood vessels [32]. To achieve the photocuring deeper within tissue, methods and devices need to be developed.

Photopolymerized poly(ethylene glycol)dimethacrylate (PEGDMA) has been widely investigated for biomedical applications such as cell encapsulation [33], tissue engineering [34] and drug delivery [35]. PEGDMA is highly hydrophilic and the resulting hydrogel properties are tunable by changing the polymer's molecular weight and water content. Cellulose fibers showed to be a promising composite material for the reinforcement of the polymeric matrices [36,37]. The use of cellulose fibers to reinforce the hydrogel matrix is advantageous because cellulose is biocompatible and its addition only slightly influences the equilibrium water content [38]. Recently, in a separate study [39], the authors have shown that the hydrogel properties such as precursor viscosity, curing kinematics, swelling ratios and mechanical characteristics (elastic modulus, fracture strain/stress, energy dissipation) can be tailored by changing the concentration of nano-fibrillated cellulose (NFC) and the PEGDM molecular weight. Furthermore it has been observed that nano-fibrillated cellulose fibers also have a positive impact onto the bio-optical scattering properties of a hydrogel which results in more efficient and homogenous curing during light illumination.

In this study, relative concentration of NFC and molecular weight of PEGDMA were tuned in the composite hydrogel in order to match closely the properties of the NP native tissue. We further hypothesize that by injecting the liquid precursor and activating it through photopolymerization, the tissue integration of the implanted material is strongly enhanced.

\section{Materials and methods}

A hydrogel was first tailored to match the properties of native NP tissue in terms of elasticity and water content. A selected hydrogel was then evaluated against native bovine NP tissue during specifically designed functional tests: 1) confined compression was done to avoid subsidence into the IVD endplate, 2) the hydrogel's swelling pressure was tested to be able to re-establish disc height and 3) an extrusion test was performed to evaluate the hydrogel's resistance to extrusion or expulsion. Following material testing, a surgical injection, illumination and monitoring device was developed and applied to implant the composite hydrogel into an ex vivo bovine IVD organ culture model.

\subsection{Sample preparation}

\subsubsection{PEGDMA synthesis}

PEGDMA was synthesized according to the description by LinGibson et al. [40]. Poly(ethylene glycol) with molecular weights of 6 and $20 \mathrm{kDa}$ and triethanolamine (99\%) were purchased from Sigma Aldrich, Buchs, Switzerland. Poly(ethylene glycol) was dried by the aid of dean-stark distillation. Extra dry dichloromethane (99.8\%) and diethyl ether (99.5\%, extra dry over molecular sieve) were purchased from Acros, Basel, Switzerland. Dried poly(ethylene glycol) (20 g) was dissolved in $60 \mathrm{ml}$ dichloromethane. Methacrylic anhydride (303 $\mathrm{mg}$ ) and triethanolamine $(462 \mathrm{mg}$ ) were added to the solution and the methacrylation was carried out under dry argon flow. After five days, the solution was precipitated in diethyl ether, filtered and dried overnight in vacuum at room temperature. The H NMR spectrum revealed a $74 \%$ and $90 \%$ degree of methacrylation for the PEGDMA 6 and $20 \mathrm{kDa}$, respectively.

\subsubsection{NFC preparation}

Cellulose pulp (bleached softwood pulp, elemental chlorine free) with a residual chlorine content of $0.4 \mathrm{wt} \%$ was purchased from Zellstoff Stendal, Arneburg, Germany. Cellulose pulp was fibrillated with a high-shear homogenizer by pumping the suspension through two consecutive chambers with diameters of 400 and $200 \mu \mathrm{m}$ (i.e., $\mathrm{H} 230 \mathrm{Z}_{400 \mu \mathrm{m}}$ and $\mathrm{H} 30 \mathrm{Z}_{200 \mu \mathrm{m}}$, respectively) for 12 passes. The resulting NFC suspension was concentrated with the aid of centrifugation $\left(5^{\prime} 000 \mathrm{rpm}, 25^{\circ} \mathrm{C}\right.$, three times during $\left.15 \mathrm{~min}\right)$. According to cryo-SEM images the NFCs diameter was in the range of $2-100 \mathrm{~nm}$ and their length in the range of a few micrometers.

\subsubsection{Composite hydrogel preparation}

Phosphate buffered saline (PBS, $\mathrm{pH} 7.4)$ was purchased from Gibco, Basel, Switzerland and 4-(2-hydro- xyethoxy) phenyl-(2hydroxy-2-propyl) ketone (Irgacure 2959) was purchased from BASF, Basel, Switzerland. PEGDMA powder (10 wt\%) and NFC (0.5 vol\%) were added to PBS. The PEGDMA was dissolved by keeping the solution in $37{ }^{\circ} \mathrm{C}$ water bath for $15 \mathrm{~min}$ and the photoinitiator Irgacure 2959 (0.1 wt\%) was added to the suspension. The suspension was then homogenized by ultra turex (IKA T25 digital, SN 25 10G, Staufen, Germany) for 20 min in a dark chamber. The homogenized suspension was degassed at a pressure of 20 mbar.

In order to characterize the hydrogel properties, the precursors were cast in plastic molds with a diameter of $8 \mathrm{~mm}$ and height of $4 \mathrm{~mm}$, covered by microscope slides and illuminated by monochromatic $365 \mathrm{~nm}$ ultra violet lamp (AxonLab, Baden, Switzerland) with an intensity of $5 \mathrm{mWcm}^{-2}$ during $30 \mathrm{~min}$.

\subsubsection{Sample sterilization}

The photoinitiator solution and the PEGDMA dissolved in PBS were passed through the $0.22 \mu \mathrm{m}$ filter (Millex ${ }^{\circledR} \mathrm{GS}$, Millipore Corporation, Bedford, MA). NFC were autoclaved and the NFC solution 
was sterilized by UV light illumination during $30 \mathrm{~min}$.

\subsubsection{Nucleus pulposus preparation}

IVDs were isolated from bovine tails (age between 10 and 14 month old) obtained from a local abattoir. The endplates were removed surgically and the discs immediately frozen. An $8 \mathrm{~mm}$ surgical biopsy punch (Stiefel, Brentford, United Kingdom) was used to extract the frozen NPs from the IVDs. To thaw the samples, they were put in a closed plastic bag during 5-10 min to avoid any evaporation of water.

\subsection{Material characterization tests}

\subsubsection{Water content evaluation}

Hydrogels and NP weights were measured after swelling in PBS $\left(\mathrm{W}_{\mathrm{S}}\right)$ and after 2 days of drying $\left(\mathrm{W}_{\mathrm{d}}\right)$ in a vacuum oven $\left(40{ }^{\circ} \mathrm{C}\right)$. The water content was then calculated from: $100 \times\left(\mathrm{W}_{\mathrm{s}}-\mathrm{W}_{\mathrm{d}}\right) / \mathrm{W}_{\mathrm{s}}$.

\subsubsection{Unconfined compression}

Mechanical properties of the swollen hydrogels and NP were characterized under displacement-controlled monotonic loading ( $1 \mathrm{~mm} / \mathrm{min}$ ) until reaching $80 \%$ compressive engineering strain of the sample. Measurements were performed with an Instron E3000 linear mechanical testing machine (Norwood, MA, United States). As illustrated in Fig. 1a, the samples were placed in a chamber filled with PBS during compression. A transparent substrate and a highresolution camera were employed to measure the sample's area throughout the test. With the measurement of the deformation area, the stress-strain curves were calculated. The elastic moduli were calculated as linear extrapolations of the true stress-strain curves in the linear range of $0.15-0.25$ strain.

\subsubsection{Confined compression}

A custom-made confinement setup with a porous upper and lower plate (Fig. 1b) was used to compress the samples. The used rate was $10 \mathrm{~mm} / \mathrm{min}$. The porous upper and lower plates were covered additionally with filter paper $(11 \mu \mathrm{m}$, grade 1 , Whatman, Kent, United Kingdom) in order to avoid any type of extrusion through the holes of the porous plate.

\subsubsection{Swelling pressure}

To evaluate the swelling pressure, a pre-load of $0.15 \mathrm{~N}$ was applied and the corresponding displacement represented the zero position. The chamber (Fig. 1b) was filled with PBS and the load was monitored while keeping the displacement constant. A preliminary $48 \mathrm{~h}$ test showed that all the samples reach the equilibrium pressure after $8 \mathrm{~h}$, thus all the tests were done over a period of $8 \mathrm{~h}$. The initial load of $0.15 \mathrm{~N}$ was subtracted and the swelling pressure calculated as the average pressure during the last hour of the test.

\subsubsection{Extrusion}

Samples were compressed at a rate of $1 \mathrm{~mm} / \mathrm{min}$ until they extruded through the hole in the lower plate (Fig. 1c). Plates with different diameters of $0.5,0.7,1,1.5$ and $2 \mathrm{~mm}$ were used. The maximally applied pressure before the sample's extrusion was reported as the extrusion pressure.

\subsection{Implantation of the hydrogel into an organ model}

\subsubsection{General protocol}

To test the performance of the composite hydrogel as a potential NP replacement, we used a bovine ex vivo IVD organ culture model (age of the animals: between 10 and 14 month old), which have been proven to be a adequate option $[41,42]$. Bovine caudal discs showed to be similar to human IVDs in terms of size, biomechanical behavior and biology $[43,44]$.

Fig. 2 illustrates the protocol which was used to prepare the IVD organ model and to evaluate the implanted photopolymerized composite hydrogels. The success of a NP replacement can be evaluated by measuring the height of an IVD during cyclic loading $[45,46]$. To evaluate the performance of the implant, the height of the implant was measured at four different "states": 1) healthy state (after disc isolation), 2) pre-operative state (degenerated disc), 3 ) post-operative state (repaired disc after hydrogel injection and photopolymerization) and 4) state during follow up (disc after cyclic loading). For each state, the organ height was measured by a caliper according to the method presented in the Supplementary data I. Before performing the measurement, a preconditioning was performed on each sample to assure inter-state comparability (red boxes in Fig. 2). The results were all normalized to the healthy disc state.
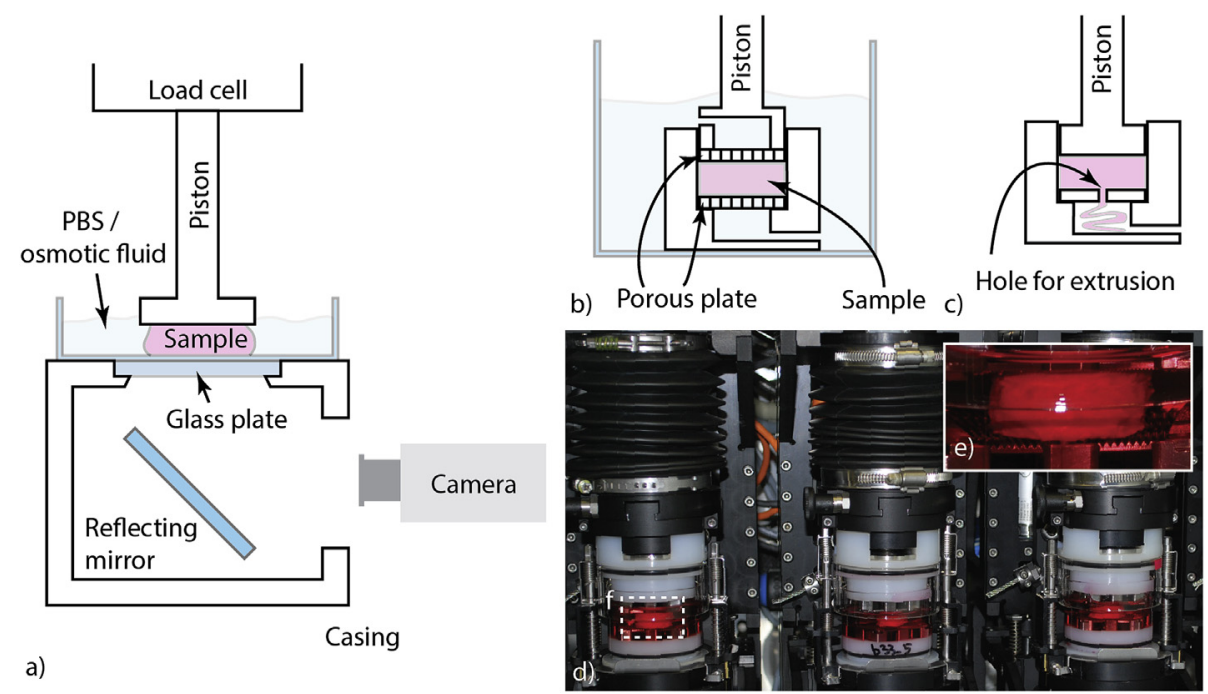

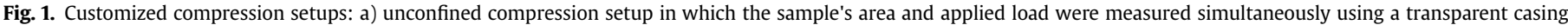

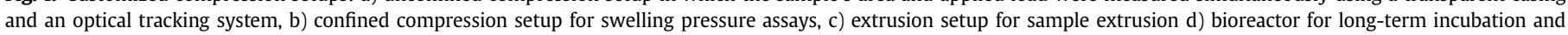
testing e) live IVD organ culture within the bioreactor chamber. 


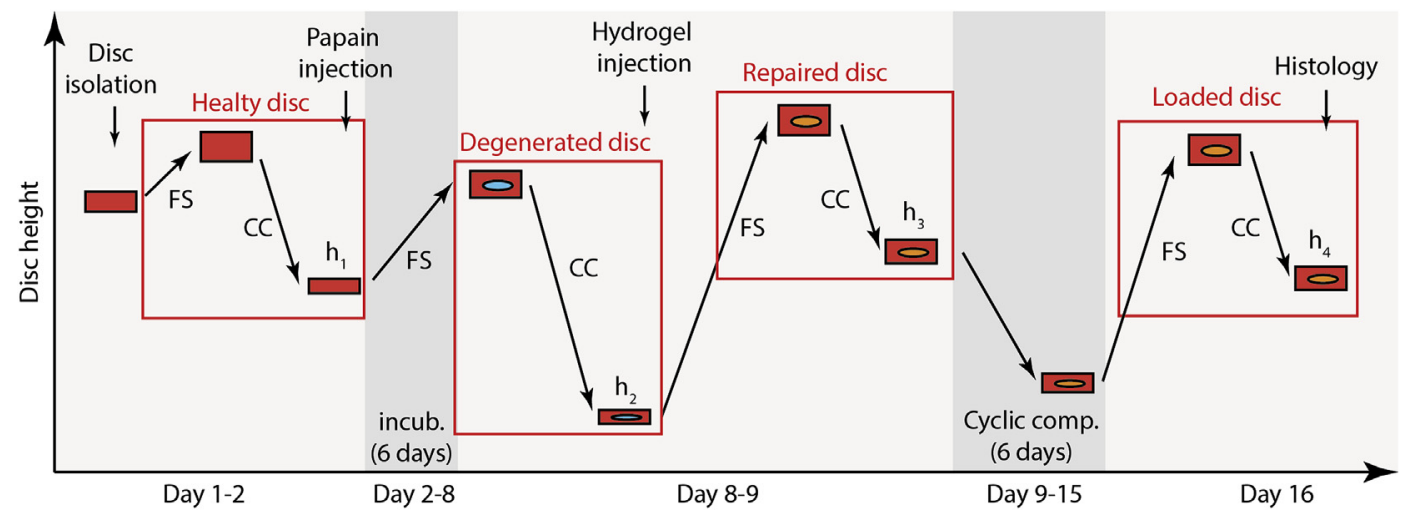

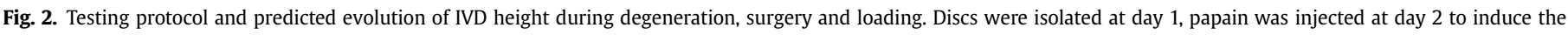

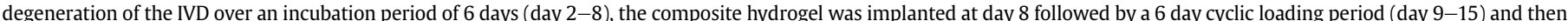

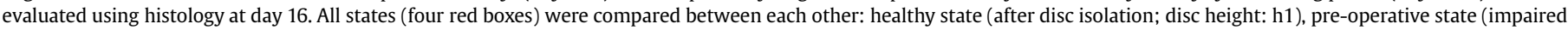

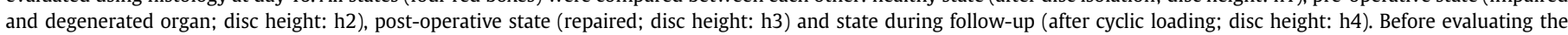

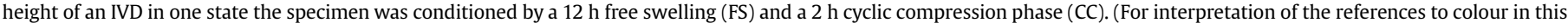
figure legend, the reader is referred to the web version of this article.)

\subsubsection{Organ model preparation}

Fresh bovine tails (animal age between 10 and 14 month old) were obtained at a local abattoir. IVDs were isolated according to the previously described protocol [41,47]. Briefly, to create the degenerated IVD model, papain (Sigma Aldrich, Buchs, Switzerland) was injected into the discs at a concentration of $100 \mathrm{U} /$ $\mathrm{ml}$. A buffer was prepared with $55 \mathrm{mM} \mathrm{Na}$-Citrate, $150 \mathrm{mM} \mathrm{NaCl}$ and $5 \mathrm{mM}$ EDTA in $400 \mathrm{ml} \mathrm{H}_{2} \mathrm{O}$. The $\mathrm{pH}$ was set to 6.0 and the volume was adjusted to $500 \mathrm{ml}$ with $\mathrm{H}_{2} \mathrm{O}$. Before injection, the papain solution was prepared by adding $5 \mathrm{mM}$ of Cysteine- $\mathrm{HCl}$ and papain $(100 \mathrm{U} / \mathrm{ml})$ to the buffer. Between 100 and $200 \mu \mathrm{l}$ of papain solution was injected via a 25 Gage needle (outer diameter $0.51 \mathrm{~mm}$ ) into each disc and the injection position was marked by a surgical thread. The injected discs were incubated $\left(37{ }^{\circ} \mathrm{C}, 5 \% \mathrm{CO}_{2}\right)$ during 6 days to digest the NP and form the cavity.

\subsubsection{Surgery device for injection, photoactivation and reaction monitoring}

To perform surgeries in a minimally invasive manner, a surgical device with the following functionalities was developed: 1) pressurization and injection of the liquid hydrogel, 2) illumination and photoactivation of the hydrogel and 3 ) reaction monitoring of the photopolymerization reaction.

This was achieved by building a customized probe which combines an injection and an illumination channel at the same time. The sample is illuminated with UVA light at $365 \mathrm{~nm}$ (photopolymerization) and visible light at $532 \mathrm{~nm}$ (monitoring). A combination of fluorescence and Raman spectroscopy is used to track the photopolymerization reaction in situ. A more extensive description of the probe and the device is presented elsewhere [48].

\subsubsection{Surgical procedure}

A 19 Gage needle (outer diameter $1.06 \mathrm{~mm}$ ) containing the optical fiber ( $600 \mu \mathrm{m}$ core, NA of 0.22 , Polymicron Technologies, Phoenix, AZ, United States) was connected to the customized probe. The area between the optical fiber and the needle was used for injection. The composite hydrogel was injected at the same location and needle path as the papain. The precursor was then illuminated during 45 min using the LED light ( $365 \mathrm{~nm}$, Nichia, Tokyo, Japan) butt-coupled to the proximal end of the optical fiber.

\subsubsection{Bioreactor loading parameters}

Each preconditioning (red boxes in Fig. 2) consisted of a $12 \mathrm{~h}$ free swelling (FS) phase and $2 \mathrm{~h}$ cyclic compression (CC) phase $(1 \mathrm{~Hz}$, $0.15 \pm 0.05 \mathrm{MPa}$ ). During the incubation phase (day 2-8) the IVDs were left under free swelling condition. During the cyclic compression phase (day 9-15) the samples were cyclically loaded $(0.2 \mathrm{~Hz}, 16 \mathrm{~h}$ per day at $0.15 \pm 0.05 \mathrm{MPa}$ and $8 \mathrm{~h}$ per day at $0.0625 \pm 0.0125 \mathrm{MPa}$ ).

\subsubsection{Histology}

Histology was done on day 16 i.e. at the end of the cyclic loading. Each IVD was cut on the transverse plain using a specifically designed cutter (Supplementary data I). After removal of the endplates by a scalpel, samples were frozen and sliced along the sagittal plain by a microtome (Leica CM3050 S, Leica Microsystems, Heerbrugg, Switzerland). Due to the high water content of the hydrogel ( $\approx 90 \mathrm{wt} \%$ ), the slice thickness was increased to $100 \mu \mathrm{m}$. Tissue sections were placed on a cover slip and fixed in 10\% formalin solution (Sigma Aldrich, Buchs, Switzerland) during 15 min. Sections were then stained with Hematoxyline \& Eosin (H\&E) solution and mounted with xylene-based glas ${ }^{\mathrm{TM}}$ medium (Sakura, Horgen, Switzerland). A light-transmitting microscope (Axiovert 100, Zeiss, Feldbach, Switzerland) was used for imaging. The magnification of the objective was 20x.

\subsection{Statistics}

All values are presented as mean ( \pm standard deviation). The statistical data analysis was done using Matlab (Mathworks, Natick MA, United States). Un-paired $t$-tests were used to compare different populations. For populations which changed over time (e.g. the organ model), paired $t$-tests were used for comparison. The population size $(\mathrm{n})$ was always between 3 and 5 samples. $\mathrm{P}<0.05$ was considered as a significant result (denoted as *). P $>0.05$ was considered as a non-significant result (ns). $\mathrm{P}<0.01$ was denoted as ** and $\mathrm{p}<0.001$ as ${ }^{* * *}$.

\section{Results}

\subsection{Hydrogel design and selection}

The material selection was done iteratively by comparing equilibrium water content, ultimate rupture strain and elastic modulus of native NP tissue and candidate hydrogels (Fig. 3). The hydrogel properties were tuned by changing the cross-linker 


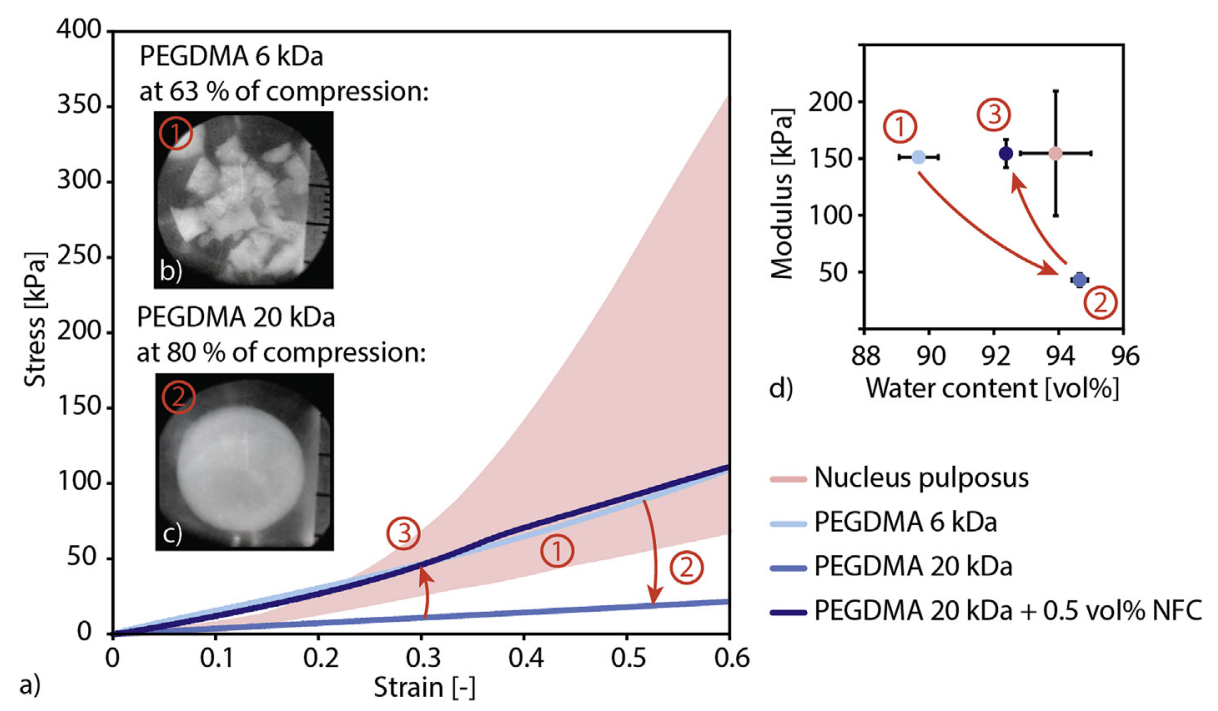

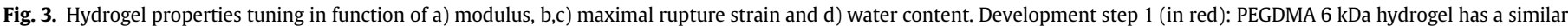

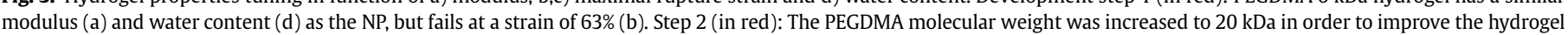

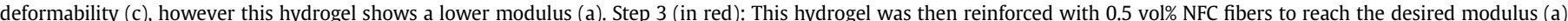

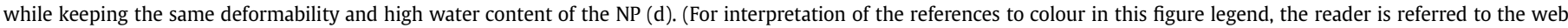
version of this article.)

density and concentration of NFC reinforcement in order to achieve high deformability and strength.

The photopolymerizable PEGDMA hydrogel with the molecular weight of $6 \mathrm{kDa}$ had a modulus and water content similar to the NP. However, this hydrogel failed under compression at a strain of $63 \%$ and a stress of $220 \mathrm{kPa}$ (Fig. 3b - development step 1). The hydrogel cross-linker density was then reduced by increasing the PEGDMA molecular weight from $6 \mathrm{kDa}$ to $20 \mathrm{kDa}$ which improved the hydrogel's ultimate rupture strain (Fig. $3 c-$ step 2 ). The resulting hydrogel was able to withstand the maximally applied strain level of $80 \%$ and had $5 \mathrm{wt} \%$ higher water content. Yet, this improvement in deformation was associated with a loss in elastic modulus of the 20 kDa PEGDMA hydrogel (Fig. 3a,d - step 2).

In order to compensate for this decrease, the material was strengthened using 0.5 vol.\%NFC, to reach the modulus of the targeted NP tissue (150 kPa), while keeping high water content (>90 wt\%). The composite hydrogel withstood the maximum applied strain of $80 \%$ without failure (Fig. 3a,d - step 3) and the precursor viscosity was kept in an injectable range (20 Pa s). Fig. 3a, illustrates the stress-strain curve of the PEGDMA $20 \mathrm{kDa}$ reinforced with $0.5 \mathrm{vol} \%$ of NFC which was used for other experiments.

\subsection{Functional testing}

The confined compressive behavior, swelling pressure and extrusion properties are specific to the organ or implant function of the IVD or the NP tissue replacement. Both native tissue and composite hydrogel were able to withstand pressures higher than 3.5 MPa in confined compression (Fig. 4a). No significant difference was observed between the composite hydrogel and the native NP tissue at each level of deformation $(0.20<\mathrm{p}<0.84)$. The equilibrium swelling pressure of the NP and the composite hydrogel were $78( \pm 8)$ and $83( \pm 6) \mathrm{kPa}$, respectively (Fig. $4 \mathrm{~b})$, however, the difference is not significant $(\mathrm{p}=0.69)$. The extrusion data illustrates that the native NP tissue was more resistant to extrusion compared to the composite hydrogel. The native NP tissue was extruded through a $0.5 \mathrm{~mm}$ hole at a pressure of $16.6( \pm 8.7) \mathrm{MPa}$, whereas the composite hydrogel was extruded at a pressure of $5.3( \pm 0.1)$ MPa. The NP tissue was always stronger: $\mathrm{p}=0.087$ (hole: $0.5 \mathrm{~mm}$ ), $\mathrm{p}=0.035(0.75 \mathrm{~mm}), \mathrm{p}=0.0000015(1 \mathrm{~mm}), \mathrm{p}=0.11(1.5 \mathrm{~mm})$ and $\mathrm{p}=0.23(2 \mathrm{~mm})$. However, the difference between the extrusion pressure was less pronounced at bigger hole sizes, e.g. a hole of $2 \mathrm{~mm}$, where the NP and the composite hydrogel were extruded at $3.2( \pm 2.4)$ and $1.2( \pm 0.05)$ MPa respectively.

\subsection{Implantation}

The extracted samples originated all from the same tail, had a diameter between 14.7 and $16.7 \mathrm{~mm}$, an initial height between 9.6 and $11.2 \mathrm{~mm}$ and weighted initially between 2.5 and $4.2 \mathrm{~g}$. The dose of papain injected was between 90 and $150 \mu$ l. The composite hydrogel was injected (Fig. 5a) at the same position and same needle path as the papain. Between 0.75 and $0.95 \mathrm{ml}$ of hydrogel could be injected per specimen. It was possible to inject the liquid hydrogel precursor through the interspace between the needle (cannula) and the optical fiber (Fig. 5b). During illumination (Fig. 5d), the photopolymerization state of the injected sample was evaluated (Fig. $5 \mathrm{c}$ ) by recording the reflected illumination spectra at different time points and by tracking the signal intensity over time. The intensity correlates directly with size of the photopolymerized hydrogel volume inside the tissue as previously shown [48]. Thus, if a known volume of hydrogel is injected, the signal intensity can be used to determine the time point of the full polymerization.

In Fig. 6, the results of the IVD loading in the bioreactor are presented. The conditioning cycles ( $12 \mathrm{~h}$ free swelling, $2 \mathrm{~h}$ cyclic compression, c.f. red boxes in Fig. 2) resulted always, as expected, in a disc height increase during the free swelling phase and a decrease during the compression phase (not shown in Fig. 6). After papain degeneration, the disc height decreased $(p=0.00022)$ to 65.6 $( \pm 0.9) \%$ of the initial, healthy disc height (Fig. 6a).

After the hydrogel injection (again including $12 \mathrm{~h}$ swelling and $2 \mathrm{~h}$ cyclic compression), the normalized disc height increased $(\mathrm{p}=0.0020)$ to $99.0( \pm 1.9) \%$. There was no significant $(\mathrm{p}=0.73)$ difference between the initial healthy disc and the repaired state. After one week of loading ( 0.5 million cycles), the disc height decreased to $91.1( \pm 6.1) \%$ of healthy initial height, i.e. the disc height suffered less than $10 \%$ height change after 0.5 million loading cycles. Compared to the degenerated state, the increase in 


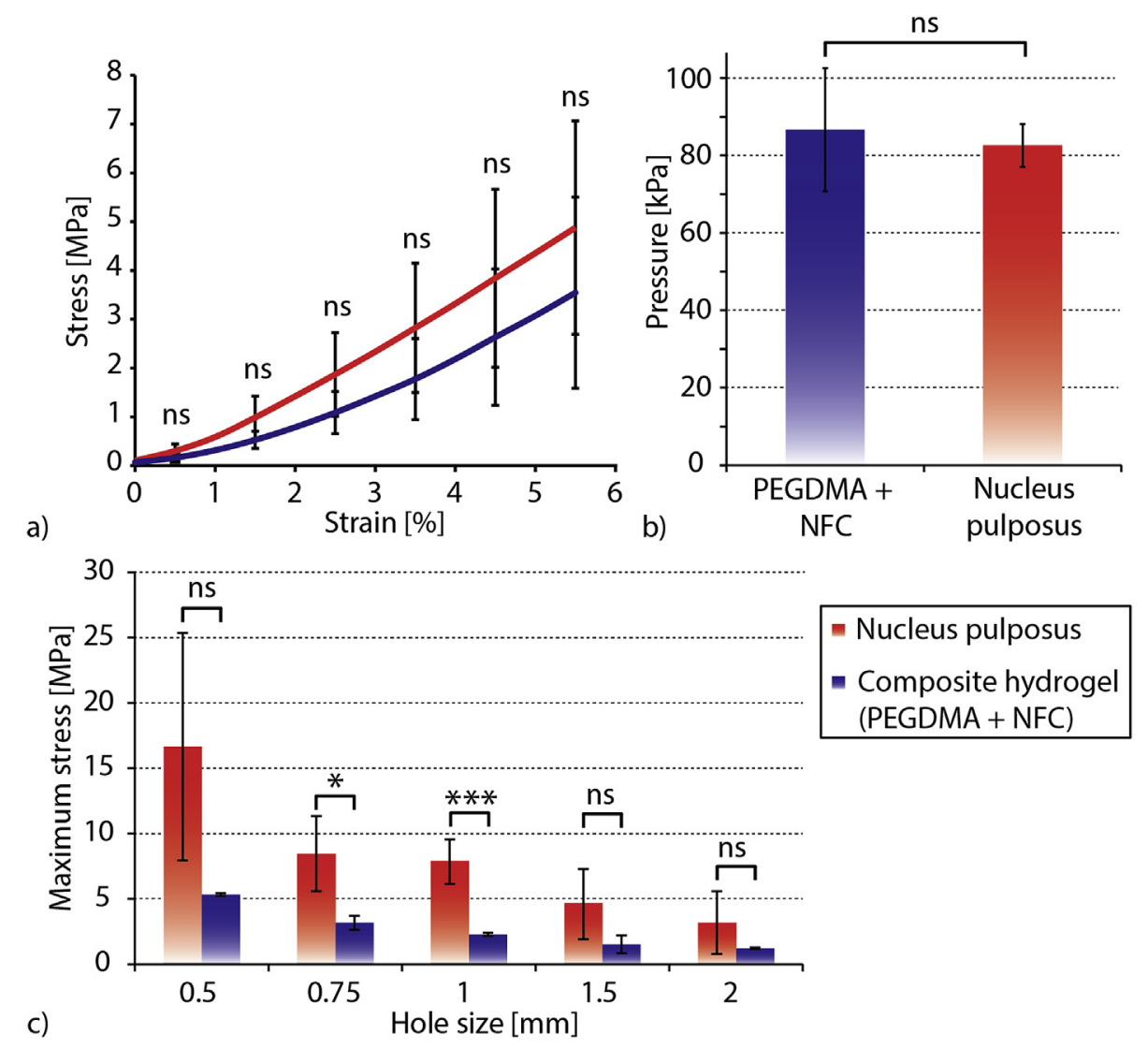

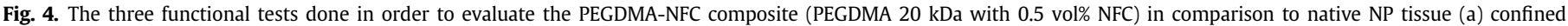

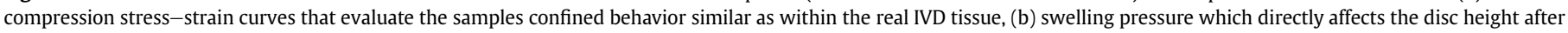
implantation and (c) the maximum tolerated stress before extrusion which assesses the sample's resistance to extrusion and expulsion.
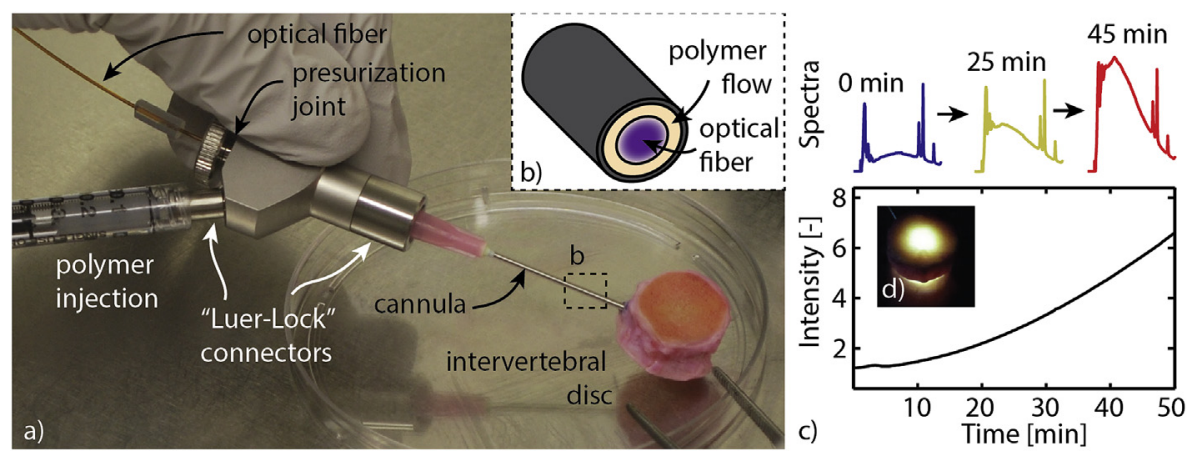

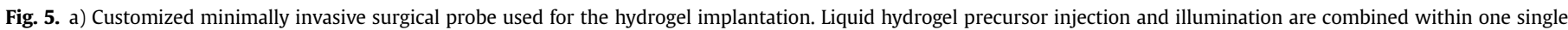

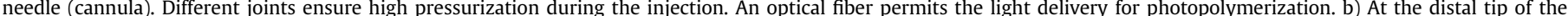

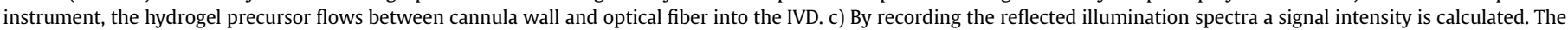

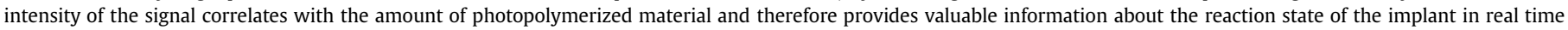
[48]. d) IVD during photopolymerization.

disc height remained significant $(p=0.021)$ and compared to the initial healthy state, no significant $(\mathrm{p}=0.11$ ) difference was found. Fig. $6 \mathrm{~b}$ and $\mathrm{c}$ illustrate graphically the increase of disc height after the surgery.

\subsection{Histology}

In Fig. $6 \mathrm{~d}$ the macroscopic integration of the composite hydrogel after loading is illustrated, surrounded by annulus fibrosus tissue and the endplates. In Fig. Ge and f, the implanted hydrogel was compared to native NP tissue (both after mechanical loading). The healthy integrity of the nuclei of the NP cells can be seen (Fig. 6e), while in Fig. $6 \mathrm{f}$ the fibrous structure of hydrogel can be observed. Fig. $6 \mathrm{~g}$ depicts the tissue integration of the hydrogel. The interface between native tissue and implanted hydrogel remained undisturbed after 0.5 million loading cycles.

\section{Discussion}

To our knowledge, this is the first study which presents the 

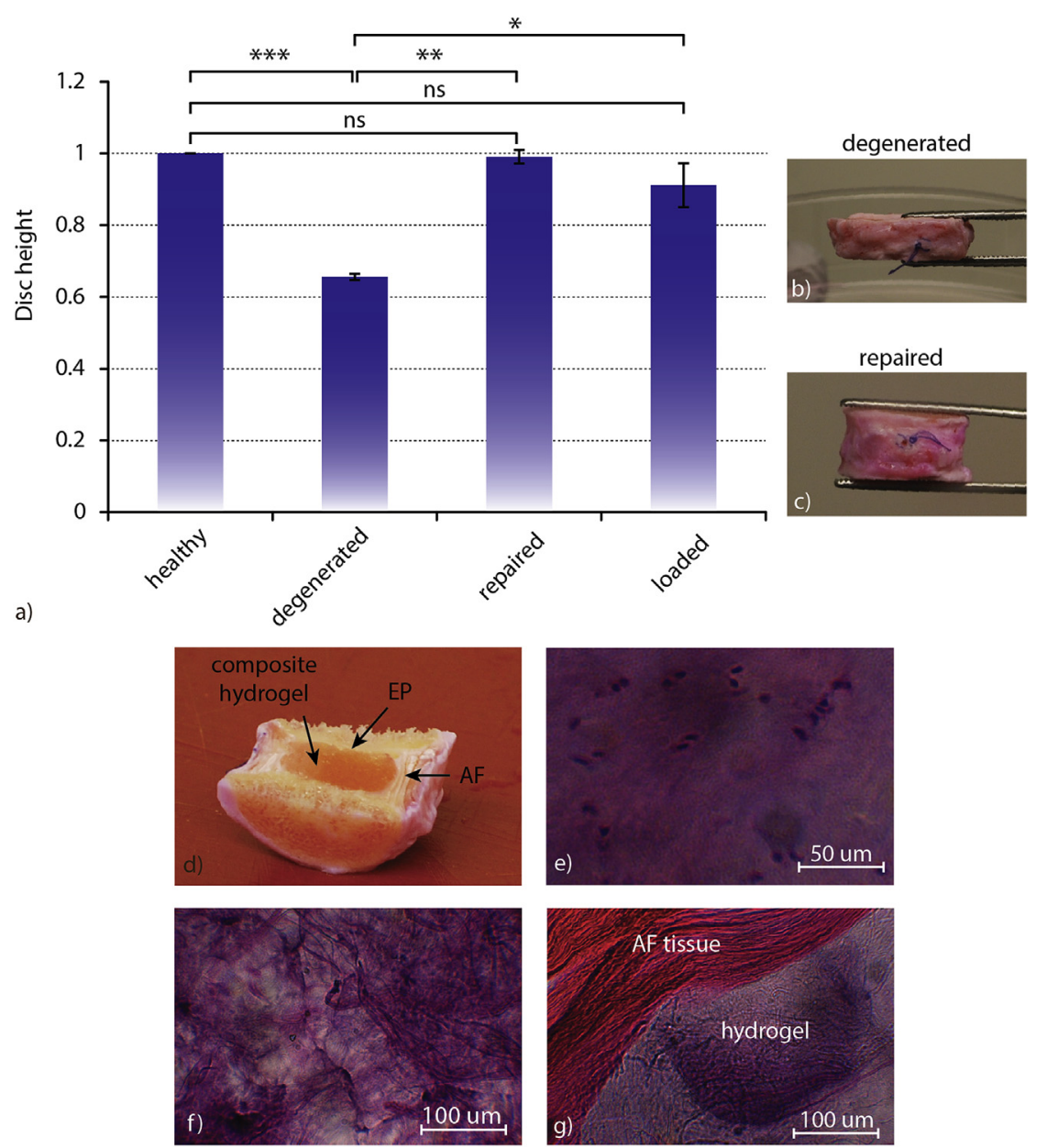

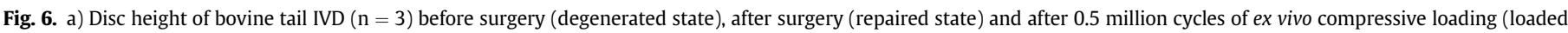

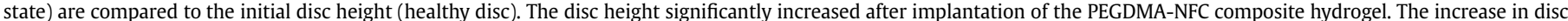

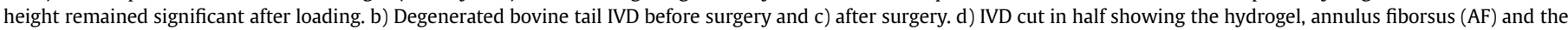

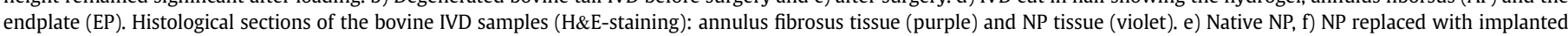

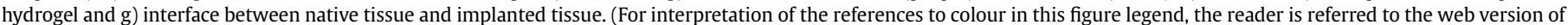
this article.)

design, implantation and testing of an in situ photopolymerized, functional orthopedic implant. A novel tuned material for NP repair was designed specifically to comply with mechanical and biooptical requirements. A device was developed to initiate and control the photo-chemical reaction in situ. The surgical procedure, medical device and implant were able to significantly restore IVD height after 0.5 million loading cycles within an ex vivo organ culture model. The hydrogel implant also showed an excellent tissue integration during histology analysis. These results not only demonstrated the feasibility of photopolymerized, mechanically loaded implants, but also open a new pathway for the minimally invasive and controlled implantation of material within the human or animal body.

Often novel materials, implantation methods and surgery devices are developed separately. In the proposed approach, the parallel development of material, surgery device and implantation method significantly contributed to the obtained promising results. To reach the goal of a working implant it was necessary to 1) tune the bio-optical and mechanical properties of the composite hydrogel, 2) design a device which allowed for in situ injection, photopolymerization and reaction monitoring and 3) evaluate the material, the device and the implantation procedure all together. The discussion will then successively focus on these three aspects.

\subsection{Material design}

\subsubsection{Mechanical properties}

Using pre-formed polymers, such as poly(ethylene glycol) instead of monomer/cross-linker systems seemed advantageous since the more homogenously formed network was stronger and showed less brittleness [25].

The composite hydrogel made of the highly deformable PEGDMA matrix reinforced with NFC had a similar modulus and water content as the healthy NP tissue. An increase of the PEGDMA's molecular weight and a decrease of the cross-linker density allowed improving the deformability of the hydrogel. The reinforcement effect provided by the NFC addition was induced by hydrogen bonds interactions between the cellulose fibrils and by the entanglement between the cellulose and the polymer networks [49]. These interactions still allowed the deformation of the networks and did not add any brittleness to the resulting composite hydrogel. The obtained composite hydrogel had a high water content owing to the hydrophilic nature of the polymer matrix and the NFCs.

The material properties were tuned in order to mimic elastic modulus and water content of native tissue while keeping the high deformability. Similar properties for the implant and the tissue result in a smooth gradient of shear stresses at the tissue/implant 
interface which reduces the risk of extrusion or expulsion. In order to evaluate the possible failure modes of the implant, different testing methods were designed. The PEGDMA-NFC composite hydrogel was tested in a confined and unconfined compression mode. The similar behavior of the composite hydrogel and the native tissue in confined compression demonstrated that the material interaction with water is similar to that of the NP tissue for the range of applied stresses and testing rates.

The comparable swelling pressures of the composite hydrogel and of the native NP indicated that the implant possibly can restore the healthy IVD height by swelling. Furthermore, the swelling lead to a continuous tissue/implant interface and ensured a homogenous load distribution. The extrusion test evaluated the hydrogel performance in the harshest condition where the material was squeezed until it ruptured completely. It could not bulge or adhere to surrounding tissue as it would do in reality. The composite hydrogel ruptured to pieces at high pressures whereas the native tissue is irreversibly deformed, but without rupturing. This different behavior might be due to a lack of available energy dissipation within the composite hydrogel system compared to the native NP tissue. This indicates that by adding energy dissipation routes in the hydrogel, e.g. via ionic interactions, an increased resistance to extrusion of the hydrogel could be expected. The extrusion test had also one contradictory aspect. There was a significant difference between NP and hydrogel with a hole of 0.75 and $1 \mathrm{~mm}$, but no significant difference for $0.5,1.5$ and $2 \mathrm{~mm}$. We do not have a clear explanation for this result. The non-signifcant difference with hole size of $0.5 \mathrm{~mm}$ might be due to the extremely high and non-physiological extrusion pressures (up to $25 \mathrm{MPa}$ ). More work is required to understand the extrusion process which might be more complex and include non linear rupture mechanisms.

\subsubsection{Bio-optical properties}

Photopolymerization allows for controlled curing and also increases the implant's adherence to native tissue. The photopolymerizable curing system is highly advantageous compared to other in situ cured systems (e.g. heat reactive or chemical curing) because it provides the surgeon with an extra degree of freedom by decoupling the material insertion and curing. Additionally, the photopolymerization of an injected, low-viscosity liquid enables the precursor to flow into tissue interstices and leads to an optimal contact between implanted material and tissue. During illumination, the penetration of liquid precursor into the microroughness of the surrounding tissue results in the formation of mechanical interlocking which improves the tissue/implant interface [50].

The illumination from an optical fiber results in a cone of light. We have previously developed a Monte Carlo model to simulate the curing light distribution in the hydrogel [51]. It was shown that the addition of light scattering elements such as intralipid particles change the conic polymerization shape to a homogenous spherical shape. However, lipid addition drastically weakened the mechanical properties of the hydrogel. To avoid this drawback, experimental data illustrated that the NFC fibers and the network they form, also act as light scattering element resulting in accelerated curing [39] and more homogeneously cured volumes [51].

\subsection{Surgical device design}

Injection, photopolymerization or monitoring of a photopolymer using a probe have been achieved previously, but the combination of the three functions in one probe is novel. We have shown that the probe can monitor the whole curing volume [48].

A potential issue of the surgical device is however the $1 \mathrm{~mm}$ incision which may lead later to the extrusion of implanted material. Within human IVDs pressures of up to 2.5 MPa have been measured [52]. The extrusion results (Fig. 4c) indicate that the hydrogel can be extruded only through holes larger than $0.5 \mathrm{~mm}$ in diameter. In order to avoid extrusion within a human IVD the probe diameter (19 Gage, $\sim 1 \mathrm{~mm}$ ) needs to be reduced to $0.5 \mathrm{~mm}$. To achieve this, a smaller optical fiber and needle would be required to do the surgery. In the current study a LED light was used for illumination. The LED can easily be replaced with laser illumination and be delivered within a small diameter fiber with an external diameter of $125 \mu \mathrm{m}$. This would allow performing the surgery through a needle as small as 25 or 26 Gages ( 0.51 and $0.46 \mathrm{~mm}$ respectively) which is a relevant factor for the delivery of hydrogels as has been shown from clinics and experimentally in animal studies [53-55].

\subsection{Evaluation of minimally invasive implantation procedure}

Usually the biomechanical evaluation of tissue is associated with high inter-sample variance and strong creep within the first $2 \mathrm{~h}$ of loading [56]. The conditioning method (free swelling and cyclic loading - c.f. red boxes in Fig. 2) counters this effect and allowed reaching standard deviations between 0.9 and $6.1 \%$ over 15 days which is low in the field [57-59]. Between day 2 and 8 the disc height decreased by $1.0 \%$ and between day 9 and 15 another $7.9 \%$. Although these changes were not significant, one could argue that ideally there should not be any decrease in disc height. However, the natural degradation of the IVD in ex vivo condition is important and usually much higher height decreases were observed over such a time frame for healthy discs [60]. Thus it can be considered that the obtained $7.9 \%$ are negligible. The histology results in Fig. $6 \mathrm{e}-\mathrm{g}$ further support these results: the in situ photopolymerized composite hydrogel was able to integrate well into existing tissue and the created interface was able to resist the extensive loading regime. This suggests that the implanted material remains at its position during loading contributing to moderate loss in disc height and low standard deviations. However, the exact adhesion or entanglement mechanisms (e.g. mechanical interlocking or covalent bonding) remain unclear and need to be further investigated.

From a general point of view, two options can be considered to evaluate the procedure and the implant: in vivo or in vitro animal models. Due to their size and metabolism, bovine, canine and ovine IVD models show high resemblance to human IVDs [61]. Yet, degraded in vivo IVD models come along with high ethical issues and limited options to control the loading. The ex vivo model in the bioreactors is an attractive solution to evaluate IVD replacements. The used bovine NP tissue has similar mechanical properties compared to human NP $[62,63]$. Additionally, bovine NP tissue can be easily obtained while human non-degenerated tissue is difficult to obtain. To obtain long-term results in vivo, an attractive option could be the canine model [64] as it is the only animal model with a clinical veterinary counterpart.

To demonstrate the potential for a clinical translation, it is required that the implant is imaged during the implantation procedure. In the Supplementary data II, the water in the hydrogel was replaced with a water-based Iodine marker. The first picture illustrated how the injected material can be imaged in a clinical situation using X-ray tomography to inject the material and visualize its position after injection. The second picture demonstrated how the T2 signal during magnetic resonance imaging could be used to differentiate between an empty and a filled cavity.

Another requirement for a clinical translation is the need to treat later stage IVD degeneration or disc herniation (which are two distinct pathologies) where the procedure is limited by the degenerative state of the AF. We show that our material is able to resist extrusion through a hole of $0.5 \mathrm{~mm}$ up to pressures of $\sim 5 \mathrm{MPa}$ and $2 \mathrm{~mm}$ up to pressures of $\sim 1 \mathrm{MPa}$ (Fig. $4 \mathrm{c}$ ). We also show that the 
material is not extruded over a cyclic loading of 0.5 million cycles in bovine intervertebral disc. Thus, the proposed implant and material bear a certain potential to treat NP and AF tissue. Yet, for pathologies including large AF fissures, it would be necessary to combine it with an AF sealant device. It should also be considered that there are other solutions such as total IVD replacement [65,66], which especially seem to be promising when imitating the viscoelastic response of the IVD [67], something which was not taken into account in this work.

A remaining challenge is to decrease the total illumination curing time, which, for this study, was chosen to be excessively long (45 $\mathrm{min}$ ) to assure optimal tissue integration. However, we demonstrated previously that volumes of more than $2 \mathrm{ml}$ (i.e. enough for a human NP) can be photopolymerized in less than 10 min using the same optical device [68]. It is also possible to choose different photoinitiators such as Eosin Y with higher photopolymerization rates in the range between $10 \mathrm{~s}$ and $3 \mathrm{~min}$ [69]. Moreover, the curing power in the experiment was limited to $4 \mathrm{~mW}$ in the fiber and can easily be increased by more than one order of magnitude using a laser light source. In summary, the procedure could easily be translated into clinics and seems to have a high potential to be successful.

\section{Conclusions}

A composite hydrogel having similar functional properties as a native bovine NP was developed. Using a specifically designed probe for in situ photopolymerization, the composite hydrogel was implanted into an IVD model and was able to withstand loading without signs of damage. The composite hydrogel showed promising performance during implantation and significantly re-established IVD disc height from 65.6 to $99.0 \%$ and maintained it on a significantly higher level over 0.5 million loading cycles during an ex vivo study. After 15 days of loading, a continuous, undisturbed tissue/implant interface was observed during histology.

\section{Acknowledgments}

Funding for this work was provided by the two SNF grants \#10024003165465 and \#310030_153411. We acknowledge Dr. T. Zimmermann and Dr. P. Tingaut (App. Wood Mat. Lab., EMPA, Dübendorf, Switzerland) for providing and preparing the NFC fibers, the group of Prof. H. Frauenrath (Lab. of Macromolec. and Organic Mat., EPFL), specifically Dr. R. Marty, for his assistance with the synthesis of PEGDMA, Sandra Jaccoud (Lab. of Biomech. Orthopedics, EPFL) for help and advice related to sample preparation and sterilization, Eva Roth (Inst. for Surg. Techn. and Biomech., University of Bern, Switzerland) for her assistance with biochemical assays and Jessica Dessimoz (Histology Core Facility, EPFL) for her help with histology. We thank Dr. Harald Bonél, Department of Diagnostic, Interventional and Pediatric Radiology, Inselspital, University of Bern for the MRI scanning of the bovine IVDs.

\section{Appendix A. Supplementary data}

Supplementary data related to this article can be found at http:// dx.doi.org/10.1016/j.biomaterials.2016.02.015.

\section{References}

[1] D. Borenstein, Mechanical low back pain[mdash]a rheumatologist's view, Nat. Rev. Rheumatol. 9 (2013) 643-653.

[2] S. Dagenais, J. Caro, S. Haldeman, A systematic review of low back pain cost of illness studies in the United States and internationally, Spine J. 8 (2008) 8-20.

[3] J.N. Katz, Lumbar disc disorders and low-back pain: socioeconomic factors and consequences, J. Bone Jt. Surg. 88 (2006) 21-24.
[4] M.W. van Tulder, B.W. Koes, L.M. Bouter, Conservative treatment of acute and chronic nonspecific low back pain: a systematic review of randomized controlled trials of the most common interventions, Spine 22 (1997) 2128-2156.

[5] G. WADDELL, 1987 Volvo award in clinical sciences: a new clinical model for the treatment of low-back pain, Spine 12 (1987) 632-644.

[6] A.H. McGregor, S.P.F. Hughes, The evaluation of the surgical management of nerve root compression in patients with low back pain: part 1: the assessment of outcome, Spine 27 (2002) 1465-1470.

[7] R. Chou, J. Baisden, E.J. Carragee, D.K. Resnick, W.O. Shaffer, J.D. Loeser, Surgery for low back pain: a review of the evidence for an American pain society clinical practice guideline, Spine 34 (2009) 1094-1109.

[8] P. Fritzell, O. Hägg, P. Wessberg, A. Nordwall, S.L.S.S. Group, 2001 Volvo award winner in clinical studies: lumbar fusion versus nonsurgical treatment for chronic low back pain: a multicenter randomized controlled trial from the swedish lumbar spine study group, Spine 26 (2001) 2521-2532.

[9] C.M. Bono, C.K. Lee, Critical analysis of trends in fusion for degenerative disc disease over the past 20 years: influence of technique on fusion rate and clinical outcome, Spine 29 (2004) 455-463.

[10] N. Miyakoshi, E. Abe, Y. Shimada, K. Okuyama, T. Suzuki, K. Sato, Outcome of one-level posterior lumbar interbody fusion for spondylolisthesis and postoperative intervertebral disc degeneration adjacent to the fusion, Spine 25 (2000) 1837-1842.

[11] M.M. Panjabi, Clinical spinal instability and low back pain, J. Electromyogr. Kinesiol. 13 (2003) 371-379.

[12] D. Coric, P.V. Mummaneni, Nucleus replacement technologies, J. Neurosurg. Spine 8 (2008) 115-120.

[13] H. Serhan, D. Mhatre, H. Defossez, C.M. Bono, Motion-preserving technologies for degenerative lumbar spine: The past, present, and future horizons, SAS J. 5 (2011) 75-89.

[14] M.H. Pelletier, W.R. Walsh, N. South, Nucleus replacement, Compr. Biomater (2011) $171-190$.

[15] J.C. Iatridis, S.B. Nicoll, A.J. Michalek, B.A. Walter, M.S. Gupta, Role of biomechanics in intervertebral disc degeneration and regenerative therapies: what needs repairing in the disc and what are promising biomaterials for its repair? spine J. off. J. North Am. Spine Soc. 13 (2013) 243-262.

[16] N.L. Nerurkar, D.M. Elliott, R.L. Mauck, Mechanical design criteria for intervertebral disc tissue engineering, J. biomech. 43 (2010) 1017-1030.

[17] S. Reitmaier, U. Wolfram, A. Ignatius, H.-J. Wilke, A. Gloria, J.M. Martín-Martínez, et al., Hydrogels for nucleus replacement-facing the biomechanical challenge, J. Mech. Behav. Biomed. Mater. 14 (2012) 67-77.

[18] G. Lewis, Nucleus pulposus replacement and regeneration/repair technologies: present status and future prospects, J. Biomed. Mater. Res. Part B, Appl. biomater. 100 (2012) 1702-1720.

[19] M. Buonocore, Adhesive sealing of pits and fissures for caries prevention, with use of ultraviolet light, J. Am. Dent. Assoc. 80 (1970) 324-328.

[20] J.A. Burdick, A.J. Peterson, K.S. Anseth, Conversion and temperature profiles during the photoinitiated polymerization of thick orthopaedic biomaterials, Biomaterials 22 (2001) 1779-1786.

[21] J.L. Hill-West, S.M. Chowdhury, M.J. Slepian, J.A. Hubbell, Inhibition of thrombosis and intimal thickening by in situ photopolymerization of thin hydrogel barriers, Proc. Natl. Acad. Sci. 91 (1994) 5967-5971.

[22] M.W. Grinstaff, Designing hydrogel adhesives for corneal wound repair, Biomaterials 28 (2007) 5205-5214.

[23] K.T. Nguyen, J.L. West, Photopolymerizable hydrogels for tissue engineering applications, Biomaterials 23 (2002) 4307-4314.

[24] K.S. Anseth, C.N. Bowman, L. Brannon-Peppas, Mechanical properties of hydrogels and their experimental determination, Biomaterials 17 (1996) 1647-1657.

[25] S. Naficy, H.R. Brown, J.M. Razal, G.M. Spinks, P.G. Whitten, Progress toward robust polymer hydrogels, Aust. J. Chem. 64 (2011) 1007-1025.

[26] G. Lake, A. Thomas, The strength of highly elastic materials, Proc. R. Soc. Lond. Ser. A Math. Phys. Sci. 300 (1967) 108-119.

[27] K.S. Anseth, C.N. Bowman, L. Brannon-Peppas, Mechanical properties of hydrogels and their experimental determination, Biomaterials 17 (1996) 1647-1657.

[28] C.W. Peak, J.J. Wilker, G. Schmidt, A review on tough and sticky hydrogels, Colloid Polym. Sci. 291 (2013) 2031-2047.

[29] Y. Okumura, K. Ito, The polyrotaxane gel: a topological gel by figure-of-eight cross-links, Adv. Mater. 13 (2001) 485-487.

[30] J.P. Gong, Y. Katsuyama, T. Kurokawa, Y. Osada, Double-network hydrogels with extremely high mechanical strength, Adv. Mater. 15 (2003) 1155-1158.

[31] J. Elisseeff, K. Anseth, D. Sims, W. McIntosh, M. Randolph, R. Langer, Transdermal photopolymerization for minimally invasive implantation, Proc. Natl. Acad. Sci. 96 (1999) 3104-3107.

[32] J.A. Hubbell, In situ material transformations in tissue engineering, MRS Bull 21 (1996) 33-35.

[33] S.J. Bryant, K.S. Anseth, Hydrogel properties influence ECM production by chondrocytes photoencapsulated in poly (ethylene glycol) hydrogels, J. Biomed. Mater. Res. 59 (2002) 63-72.

[34] S.J. Bryant, K.S. Anseth, Controlling the spatial distribution of ECM components in degradable PEG hydrogels for tissue engineering cartilage, J. Biomed. Mater. Res. Part A 64 (2003) 70-79.

[35] N. Nagai, H. Kaji, H. Onami, Y. Ishikawa, M. Nishizawa, N. Osumi, et al. A polymeric device for controlled transscleral multi-drug delivery to the 
posterior segment of the eye, Acta Biomater. 10 (2014) 680-687.

[36] I. Siró, D. Plackett, Microfibrillated cellulose and new nanocomposite materials: a review, Cellulose 17 (2010) 459-494.

[37] T. Zimmermann, E. Pöhler, T. Geiger, Cellulose fibrils for polymer reinforcement, Adv. Eng. Mater. 6 (2004) 754-761.

[38] A.C. Borges, C. Eyholzer, F. Duc, P.E. Bourban, P. Tingaut, T. Zimmermann, et al, Nanofibrillated cellulose composite hydrogel for the replacement of the nucleus pulposus, Acta Biomater. 7 (2011) 3412-3421.

[39] A. Khoushabi, A. Schmocker, C. Schizas, C. Moser, D. Pioletti, J.-A. Manson, et al., The combined roles of NFC fibres and PEGDM molecular weight on photopolymerization, swelling behaviour and mechanical properties of composite hydrogels, Compos. Sci. Technol. 119 (2015) 93-99.

[40] S. Lin-Gibson, S. Bencherif, J.A. Cooper, S.J. Wetzel, J.M. Antonucci, B.M. Vogel, et al., Synthesis and characterization of peg dimethacrylates and their hydrogels, Biomacromolecules 5 (2004) 1280-1287.

[41] S.C.W. Chan, A. Bürki, H.M. Bonél, L.M. Benneker, B. Gantenbein-Ritter, Papaininduced in vitro disc degeneration model for the study of injectable nucleus pulposus therapy, Spine J. off. J. North Am. Spine Soc. 13 (2013) 273-283.

[42] B. Gantenbein, S. Illien-Jünger, S.C. Chan, J. Walser, L. Haglund, S.J. Ferguson, et al., Organ culture bioreactors-platforms to study human intervertebral disc degeneration and regenerative therapy, Curr. stem cell Res. Ther. 10 (2015) 339.

[43] J.C. Beckstein, S. Sen, T.P. Schaer, E.J. Vresilovic, D.M. Elliott, Comparison of animal discs used in disc research to human lumbar disc: axial compression mechanics and glycosaminoglycan content, Spine 33 (2008) E166-E173.

[44] C.N. Demers, J. Antoniou, F. Mwale, Value and limitations of using the bovine tail as a model for the human lumbar spine, Spine 29 (2004) 2793-2799.

[45] C.L. Korecki, J.J. Costi, J.C. Iatridis, Needle puncture injury affects intervertebral disc mechanics and biology in an organ culture model, Spine 33 (2008) 235-241.

[46] S. Reitmaier, L. Kreja, K. Gruchenberg, B. Kanter, J. Silva-Correia, J. Oliveira, et al., In vivo biofunctional evaluation of hydrogels for disc regeneration, Eur. Spine J. 23 (2014) 19-26.

[47] C.W.C. Samantha, G.-R. Benjamin, Preparation of intact bovine tail intervertebral discs for organ culture, J. Vis. Exp. (60) (2012).

[48] A. Schmocker, A. Khoushabi, C. Schizas, P.-E. Bourban, D. Pioletti, C. Moser Miniature probe for the delivery and monitoring of a photopolymerizable material, J. Biomed. Opt. 20 (12) (2015).

[49] M.-P. Lowys, J. Desbrieres, M. Rinaudo, Rheological characterization of cellulosic microfibril suspensions. Role of polymeric additives, Food Hydrocoll. 15 (2001) 25-32.

[50] J. Elisseeff, K. Anseth, D. Sims, W. McIntosh, M. Randolph, R. Langer, Transdermal photopolymerization for minimally invasive implantation, Proc. Natl Acad. Sci. 96 (1999) 3104-3107.

[51] A. Schmocker, A. Khoushabi, C. Schizas, P.-E. Bourban, D.P. Pioletti, C. Moser, Photopolymerizable hydrogels for implants: MONTE-Carlo modeling and experimental in vitro validation, J. Biomed. Opt. 19 (2014) 35004.

[52] H.J. Wilke, P. Neef, M. Caimi, T. Hoogland, L.E. Claes, New in vivo measurements of pressures in the intervertebral disc in daily life, Spine 24 (1999) $755-762$.

[53] D.M. Elliott, C.S. Yerramalli, J.C. Beckstein, J.I. Boxberger, W. Johannessen, E.J. Vresilovic, The effect of relative needle diameter in puncture and sham injection animal models of degeneration, Spine 33 (2008) 588-596.
[54] J.-L. Wang, Y.-C. Tsai, Y.-H. Wang, The leakage pathway and effect of needle gauge on degree of disc injury post anular puncture: a comparative study using aged human and adolescent porcine discs, Spine 32 (2007) 1809-1815.

[55] E.J. Carragee, A.S. Don, E.L. Hurwitz, J.M. Cuellar, J. Carrino, R. Herzog, 2009 ISSLS prize winner: does discography cause accelerated progression of degeneration changes in the lumbar disc: a ten-year matched cohort study, Spine 34 (2009) 2338-2345

[56] M.A. Adams, D.W. McMillan, T.P. Green, P. Dolan, Sustained loading generates stress concentrations in lumbar intervertebral discs, Spine 21 (1996) 434-438.

[57] R.D. Bowles, H.H. Gebhard, R. Hartl, L.J. Bonassar, Tissue-engineered intervertebral discs produce new matrix, maintain disc height, and restore biomechanical function to the rodent spine, Proc. Natl. Acad. Sci. 108 (2011).

[58] N. Bergknut, La Smolders, L.H. Koole, G. Voorhout, R.E. Hagman, A.S. Lagerstedt, et al., The performance of a hydrogel nucleus pulposus prosthesis in an ex vivo canine model, Biomaterials 31 (2010) 6782-6788.

[59] K. Wuertz, K. Godburn, J.J. MacLean, A. Barbir, J. Stinnett Donnelly, P.J. Roughley, et al., In vivo remodeling of intervertebral discs in response to short-and long-term dynamic compression, J. Orthop. Res. 27 (2009) 1235-1242.

[60] C. Korecki, J. MacLean, J. Iatridis, Characterization of an in vitro intervertebral disc organ culture system, Eur. Spine J. 16 (2007) 1029-1037.

[61] M. Alini, S.M. Eisenstein, K. Ito, C. Little, Masuda K. Kettler aA, et al., Are animal models useful for studying human disc disorders/degeneration? Eur. Spine J. off. Publ. Eur. Spine Soc. Eur. Spinal Deform. Soc. Eur. Sect. Cerv. Spine Res. Soc. 17 (2008) 2-19.

[62] W. Johannessen, D.M. Elliott, Effects of degeneration on the biphasic material properties of human nucleus pulposus in confined compression, Spine 30 (2005).

[63] D. Périé, D. Korda, J.C. Iatridis, Confined compression experiments on bovine nucleus pulposus and annulus fibrosus: sensitivity of the experiment in the determination of compressive modulus and hydraulic permeability, J. biomech. 38 (2005) 2164-2171.

[64] N. Bergknut, J.P. Rutges, H.-J.C. Kranenburg, L.A. Smolders, R. Hagman, H.J. Smidt, et al., The dog as an animal model for intervertebral disc degeneration? Spine 37 (2012) 351-358.

[65] A. Gloria, F. Causa, R. De Santis, P.A. Netti, L. Ambrosio, Dynamic-mechanical properties of a novel composite intervertebral disc prosthesis, J. Mater. Sci. Mater. Med. 18 (2007) 2159-2165.

[66] A. Gloria, R. De Santis, L. Ambrosio, F. Causa, K.E. Tanner, A multi-component fiber-reinforced PHEMA-based hydrogel/HAPEXTM device for customized intervertebral disc prosthesis, J. Biomater. Appl. 25 (2011) 795-810.

[67] J. Silva-Correia, A. Gloria, M.B. Oliveira, J.F. Mano, J.M. Oliveira, L. Ambrosio, et al., Rheological and mechanical properties of acellular and cell-laden methacrylated gellan gum hydrogels, J. Biomed. Mater. Res. Part A 101 (2013) 3438-3446.

[68] A.M. Schmocker, A. Khoushabi, B. Gantenbein-Ritter, S. Chan, H.M. Bonél, P.E. Bourban, et al., Minimally invasive photopolymerization in intervertebral disc tissue cavities, in: A. Wax, V. Backman (Eds.), SPIE BiOS: International Society for Optics and Photonics, 2014, p. 895206.

[69] Y. An, Ja Hubbell, Intraarterial protein delivery via intimally-adherent bilayer hydrogels, J. Control. Release 64 (2000) 205-215. 